\section{Ocorrências em pronto-socorro: eventos sentinela para o monitoramento da atenção básica de saúde}

\author{
Caseload profile in emergency services: sentinel \\ events for monitoring primary health care
}

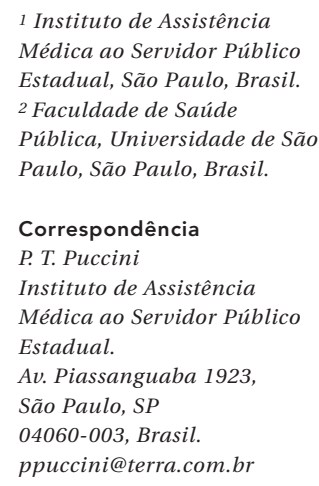

\section{Abstract}

The study's hypothesis was that certain disease entities treated in emergency services, based on the sentinel event concept, are related to flaws in primary care and can be used to develop an indicator for monitoring such care. Quantitative and qualitative data were used on previously selected occurrences treated in the emergency department. Using triangulation of methods, the differences were verified in the frequencies of these occurrences between the areas of patients' residencel primary care units and the motivations leading them to seek the emergency department. Distribution of the frequency of these events varied from $30 \%$ to $42.8 \%$, according to the area of residencel primary care unit $\left(\chi^{2}=\right.$ 9.19 and $p=0.027$ ). The interviews suggested the existence of causality between the reason stated for seeking the emergency service and the performance of the primary care units. In conclusion: (1) the frequency of the selected occurrences was influenced by the area of residencel location of the primary care unit; (2) this influence results partially from the primary care performance; and (3) the study instrument is simple and can help local administrators monitor the daily performance of primary care services.

Sentinel Surveillance; Health Services Evaluation; Primary Health Care
Paulo de Tarso Puccini 1

Vitória Kedy Cornetta 2

\section{Introdução}

A reorganização da rede básica de saúde representa um processo fundamental na reforma do setor saúde e é, dentre outras questões, dependente da capacidade de gestão. Avaliação e monitoramento são etapas essenciais do processo de planejamento e administração. São componentes dessa capacidade de gestão que possibilitam a tomada de decisão de maneira mais racional e atenta aos problemas enfrentados, viabilizando um contexto democrático para a participação e o controle social 1,2 .

Objetivando orientar o processo de implementação e avaliação da atenção básica, o Ministério da Saúde formulou a proposta do Pacto de Indicadores da Atenção Básica ${ }^{3}$. Reconhecendo a potencialidade desse pacto de indicadores, $\mathrm{Me}$ dina et al. 4 destacam, entretanto, a necessidade de novas ferramentas para conferir agilidade ao processo de monitoramento e maior possibilidade de direcionamento das ações em tempo real. Com as mudanças na rede básica evidenciase, portanto, a necessidade de instrumentos de apoio ao monitoramento que possam contribuir para a gestão local 5 . Nos últimos anos vem se delineando uma política de institucionalização da avaliação na atenção básica, entendida como uma estratégia com o objetivo de incorporá-la no cotidiano dos gestores e profissionais 6 , buscando superar a concepção de uma prestação de contas a organismos financiadores 7 . Hartz 8 
enfatiza as diferenças entre as atividades de avaliação e as de monitoramento, afirmando que o monitoramento estaria mais focado na geração de informações de fácil apropriação e utilidade para o dia-a-dia dos gestores, para a tomada de decisão e aperfeiçoamento dos programas.

Em processos de monitoramento, tem sido freqüente o uso de eventos sentinela, que na gestão de serviços de saúde são conceituados como algo que não deveria ocorrer se os serviços de saúde funcionassem adequadamente 9 . Com base nesse conceito de eventos sentinela, o objetivo do presente trabalho foi verificar se determinadas ocorrências nosológicas atendidas em prontossocorros, previamente escolhidas como ocorrências negativas, poderiam ser relacionadas com possíveis falhas ou insuficiências dos serviços básicos de saúde e, assim, converterem-se em indicadores que contribuam com a gestão local no monitoramento da rede básica de saúde.

\section{Método}

Estudo transversal realizado em 2004, no Município de Itapecerica da Serra, localizado na região sudoeste da Grande São Paulo, Brasil, com população estimada de 147.540 habitantes. O município contava com dez unidades básicas de saúde (UBS), um ambulatório de especialidades, dois prontos-socorros, uma maternidade e um hospital geral, além de serviços de controle de zoonoses, vigilância sanitária e epidemiológica. Foram escolhidas para o estudo quatro UBS com diferentes portes, cuja população $(58,6 \%$ do total do município) utilizava, preferencialmente, o Pronto-socorro Central de Itapecerica da Serra como referência. As populações das áreas de abrangência dessas unidades e respectivas porcentagens em relação ao total do município eram: UBS A: 36.500 (24,7\%), UBS B: 24.500 (16,6\%), UBS C: $18.500(12,5 \%)$ e UBS D: $7.000(4,7 \%)$. Todas as unidades atuavam segundo o modelo tradicional de assistência, contando com clínicos, pediatras e gineco-obstetras.

Avaliar é estabelecer um juízo de valor, pressupõe um posicionamento do avaliador e do avaliado sobre o que avaliar e o conteúdo das conclusões 10. Não é um processo exclusivamente técnico, um método pelo qual, dispondo-se de medidas se possa deduzir, quase que algebricamente, a efetividade de determinado serviço ou programa. Avaliar é também decodificar os interesses em disputa, visando a um entendimento contextualizado da cultura institucional e da prática dos agentes que o serviço ou programa envolve 11. Considerando esse entendimento metodológico, o estudo desenvolveu-se com base em dados quantitativos e qualitativos a fim de explorar de maneira mais aprofundada as condições de ocorrência de alguns tipos de atendimentos realizados no pronto-socorro. Orientaram a elaboração dos instrumentos de coleta de dados para um monitoramento da atenção básica os princípios que mais se articulam com as transformações pretendidas pela reforma brasileira: acesso, integralidade com humanização dos serviços, conceito ampliado de saúde e participação da população 12,13.

A coleta de dados compreendeu, portanto: (1) características dos atendimentos realizados no Pronto-socorro Central de Itapecerica da Serra (sexo, idade, área UBS de procedência e morbidade/Classificação Internacional de Doenças, 10a revisão - CID-10 14) durante duas semanas, de 2 a a $6 \underline{a}$, das 7 às 16 horas (obtidas por meio das fichas de atendimento ambulatorial); (2) entrevistas (amostragem por quotas) com as pessoas atendidas no pronto-socorro com uma das hipóteses diagnósticas escolhidas, realizadas usandose um formulário que indagava sobre: identificação do paciente, tipo de uso rotineiro de unidade básica, problema atual (queixa e tempo de queixa), procura ou não de atendimento anterior até a decisão de procurar o pronto-socorro pelo problema atual, e o discurso em relação ao atendimento ocorrido na unidade básica pelo problema atual antes de procurar o pronto-socorro ou por que não procurou a unidade básica pelo problema atual; (3) observação das características da atuação das UBS, coletadas utilizandose um formulário por técnicos da secretaria da saúde do município. As informações obtidas por esse formulário incluíam: população adscrita por UBS, cobertura referente às ações programáticas (pré-natal, puericultura, vacinação e outras), constituição da equipe de profissionais de saúde, características relacionadas ao acesso e organização da assistência (acolhimento, agendamento, atuação da enfermagem), grupos educativos, ações na comunidade, existência e atuação de conselho gestor na unidade. Neste trabalho foram consideradas somente as características referentes ao acesso e organização da assistência.

A escolha das ocorrências atendidas no pronto-socorro utilizadas no estudo foi realizada numa etapa preliminar do trabalho. O julgamento para a inclusão ou não de determinada ocorrência realizou-se segundo os critérios: (1) freqüência da indicação pelos médicos socorristas das ocorrências, segundo área básica de atendimento (pediatria, mulher, adulto), que poderiam ter sido atendidas na UBS - esta indicação foi coletada por meio de entrevista dirigida por questionário; (2) freqüência da ocorrência verificada no levantamento piloto no pronto- 
socorro: retiraram-se aquelas ocorrências que, mesmo indicadas pelos médicos socorristas e consideradas como características do papel da atenção básica, não se apresentaram no levantamento piloto numa freqüência mínima para o estudo, isto é, quando foram menores que $0,8 \%$ do total dos atendimentos considerados - este cálculo visava a que uma determinada ocorrência escolhida pudesse ter chance de estar representada com ao menos um caso em todas as áreas/UBSs estudadas (amigdalite, nasofaringite, otite média, sinusite, hipertensão); (3) possibilidade de monitorar dois aspectos da mudança em curso da rede básica local, de interesse do gestor local, mesmo sendo baixa a freqüência da ocorrência verificada no levantamento piloto, sendo eles: (3.1) a efetivação do cuidado continuado em cada UBS (puericultura e diabetes); (3.2) a ampliação da integralidade do cuidado, diferenciando estágios da dinâmica resolutiva de cada unidade básica ao incorporar novas doenças não acompanhadas até recentemente (asma brônquica, distúrbios e irregularidades da menstruação e vulvovaginites).

As ocorrências assim escolhidas são apresentadas na Tabela 1, ordenadas nas três áreas básicas de cuidados. Esse conjunto de ocorrências adotado não tem a pretensão de ser o único possível, pelo contrário, o entendimento metodológico é que seja importante adotar em cada local um processo de escolha flexível, intencionalmente relacionado à situação a ser estudada. Esta é uma estratégia necessária na definição do que comporá ou não o rol de ocorrências, para que ele seja o mais discriminatório possível, em correspondência à realidade local de uma situação da atenção básica a ser monitorada.

Para examinar os discursos das pessoas atendidas no pronto-socorro, utilizou-se a técnica de análise de conteúdo segundo categorias temáticas. De acordo com Bardin 15, esse tipo de análise realiza operações de desmembramento do texto em unidades de significado, segundo categorias preestabelecidas e, embora limitada, essa é uma técnica rápida, eficaz e que pode ser aplicada a discursos diretos e simples, como é o presente caso. Consiste em identificar núcleos de sentido presentes na enunciação, descrevê-los quanto ao significado temático e à freqüência, tendo como pressuposto que estes núcleos podem significar aspectos esclarecedores para o objetivo do estudo 11,15. Assim, a coleta dos discursos das pessoas atendidas viabiliza, na exploração retrospectiva da ocorrência, a incorporação de elementos da percepção dos usuários sobre os serviços e seus problemas. No processo de análise, as unidades de significação foram gradativamente definidas com a exploração das particularidades do material empírico coletado, e segundo a orientação propiciada pelos mesmos princípios que orientaram a elaboração dos instrumentos de coleta de dados. As categorias temáticas foram definidas com a aproximação entre o sentido principal do material empírico interpretado segundo os princípios norteadores e as questões operacionais percebidas pelas pessoas.

Tabela 1

Ocorrências atendidas em pronto-socorro, escolhidas como possíveis indicadores para o monitoramento da atenção básica. Itapecerica da Serra, São Paulo, Brasil 2004

\begin{tabular}{|c|c|c|}
\hline Área & CID-10 & Ocorrência \\
\hline \multirow[t]{5}{*}{ Comum } & $\mathrm{J} 03$ & Amidalite \\
\hline & $\mathrm{J} 45$ & Asma brônquica \\
\hline & $\mathrm{J} 00$ e J11 & Nasofaringite \\
\hline & $\mathrm{H} 66$ & Otite média \\
\hline & J01 & Sinusite \\
\hline Clínica pediátrica & ZOO e L22 & $\begin{array}{l}\text { Puericultura: problemas relacionados à antropometria, } \\
\text { cólica do recém-nascido, obstrução nasal do lactente, } \\
\text { amamentação e dermatite das fraldas }\end{array}$ \\
\hline \multirow[t]{2}{*}{ Clínica do adulto } & E14 & Diabetes \\
\hline & 110 & Hipertensão arterial sistêmica \\
\hline \multirow[t]{2}{*}{ Clínica da mulher } & N91, N92, N94 & Distúrbios e irregularidades da menstruação \\
\hline & N76 & Vulvovaginites \\
\hline
\end{tabular}

CID-10: Classificação Internacional de Doenças, 10ạ revisão 14. 
Foram quatro as etapas de organização e análise dos dados: (1) verificar a existência de diferença entre as freqüências de hipóteses diagnósticas escolhidas de cada área de procedência: uso do conceito de chance; (2) verificar a significância estatística das diferenças entre os índices das áreas/UBS: (a) em seu conjunto com o uso do teste do qui-quadrado e (b) entre pares de áreas / UBS com o uso do odds ratio (OR); (3) conhecer os discursos das pessoas atendidas: análise de conteúdo por categorias temáticas; (4) verificar as características da atuação das UBS coletadas com observação dos serviços guiada por formulário.

Para as análises estatísticas foram utilizados os programas Excel 2000 (Microsoft Corp., Estados Unidos) e SPSS versão 10.0 (SPSS Inc., Chicago, Estados Unidos). O nível de confiança utilizado foi de $95 \%$. A pesquisa e os instrumentos usados foram previamente aprovados por Comitê de Ética em Pesquisa e pelo gestor municipal de saúde.

\section{Resultados}

A distribuição dos pacientes atendidos, com uma das hipóteses diagnósticas escolhidas no Prontosocorro Central de Itapecerica da Serra, em relação às variáveis sexo e dia da semana de coleta dos dados não apresentou diferenças significantes, segundo as áreas/UBS de procedência. Para controlar os vieses ocasionados pelo tamanho da população de cada área e pela proximidade maior ou menor de cada área/UBS com a unidade socorrista, foi utilizado o índice resultante da razão hipóteses diagnósticas escolhidas/outras hipóteses diagnósticas, de cada área. Esse índi- ce expressa a chance de ocorrência de hipótese diagnóstica escolhida em cada área/UBS, que em termos probabilísticos independe do tamanho da população de cada área ou da maior ou menor procura geral desse pronto-socorro por estar geograficamente mais próxima ou mais distante dele.

\section{1ạ Etapa}

Em novembro de 2004, no período de dez dias de coleta de dados, de segunda a sexta-feira, das 7 às 16 horas e considerando as áreas de procedência das pessoas segundo o interesse do estudo, foram registrados 1.687 atendimentos, sendo 625 casos de hipóteses diagnósticas escolhidas e 1.062 de outras hipóteses diagnósticas. As freqüências das hipóteses diagnósticas escolhidas variaram significativamente $(\mathrm{p}=0,027)$ conforme a área/UBS de origem das pessoas atendidas (Tabela 2).

\section{2a Etapa}

Comparadas em pares, as quatros áreas/UBS apresentaram diferença da chance de ocorrência de hipótese diagnóstica escolhida (OR) estatisticamente significante em três das seis comparações (Tabela 3). A maior diferença foi verificada entre a área/UBS D e área/UBS C - OR = 1,75 (IC95\%: 1,15-2,65).

\section{3a Etapa}

Foram 113 pessoas entrevistadas dentre as 625 atendidas por uma das hipóteses diagnósticas escolhidas e, embora a amostragem tenha sido não probabilística por quotas, não houve diferença estatisticamente significante da amostra

Tabela 2

Freqüência das hipóteses diagnósticos escolhidas e outras hipóteses diagnósticos, no horário-unidade básica de saúde (UBS) *, segundo áreas/UBSs de procedência dos usuários. Pronto-socorro Central de Itapecerica da Serra, São Paulo, Brasil, 2004.

\begin{tabular}{|c|c|c|c|c|c|c|}
\hline \multirow[t]{2}{*}{ Área/UBS } & \multicolumn{2}{|c|}{ Hipóteses diagnósticos escolhidas } & \multicolumn{2}{|c|}{ Outras hipóteses diagnósticos } & \multicolumn{2}{|c|}{ Total } \\
\hline & $\mathbf{n}$ & $\% * *$ & $\mathbf{n}$ & $\% * \star$ & $\mathbf{n}$ & $\%$ ** \\
\hline A & 308 & 38,6 & 489 & 61,4 & 797 & 100,0 \\
\hline B & 169 & 37,1 & 286 & 62,9 & 455 & 100,0 \\
\hline C & 89 & 30,0 & 208 & 70,0 & 297 & 100,0 \\
\hline $\mathrm{D}$ & 59 & 42,8 & 79 & 57,2 & 138 & 100,0 \\
\hline Total & 625 & 37,0 & 1.062 & 63,0 & 1.687 & 100,0 \\
\hline
\end{tabular}

* 7 às 16 horas;

** Porcentagem calculada com exclusão dos ignorados, que totalizam 24 (1,4\% do total).

$\chi^{2}=9,19$ e valor de $p=0,027$. 
Verificação da influência das áreas/unidades básicas de saúde (UBS) de origem dos atendimentos sobre a freqüência das hipóteses diagnósticos escolhidas, segundo pares de UBS. Pronto-socorro Central de Itapecerica da Serra, São Paulo, Brasil, 2004.

\begin{tabular}{|c|c|c|c|c|}
\hline \multirow[t]{2}{*}{ Pares de UBS } & \multicolumn{4}{|c|}{ Hipóteses diagnósticos escolhidas-Outras hipóteses diagnósticos } \\
\hline & $\chi^{2}$ & Valor de $p$ & OR & IC95\% \\
\hline $\mathrm{D} / \mathrm{C}$ & 6,86 & $<0,01$ & 1,75 * & $1,15-2,65$ \\
\hline $\mathrm{A} / \mathrm{C}$ & 7,05 & $<0,01$ & 1,47 * & $1,11-1,96$ \\
\hline $\mathrm{B} / \mathrm{C}$ & 4,11 & 0,004 & 1,38 * & $1,01-1,53$ \\
\hline$D / A$ & 0,83 & 0,36 & 1,19 & $0,82-1,71$ \\
\hline $\mathrm{D} / \mathrm{B}$ & 1,41 & 0,24 & 1,26 & $0,86-1,86$ \\
\hline$A / B$ & 0,28 & 0,60 & 1,07 & $0,83-1,36$ \\
\hline
\end{tabular}

* Valores de odds ratio (OR) significantes para intervalo de 95\% de confiança (IC95\%).

em relação aos totais das pessoas atendidas segundo cada uma das áreas/UBS de procedência $\left(\chi^{2}=2,75 ; \mathrm{p}=0,43\right)$.

Categorizados os motivos de procura de atendimento no Pronto-socorro Central de Itapecerica da Serra por uma das ocorrências escolhidas, obtiveram-se as seguintes categorias discursivas:

\section{- Uso de assistência particular, plano privado de saúde, peregrinos}

Nesta categoria estão as motivações das pessoas que não usam regularmente a UBS por terem médico particular ou convênio, ou são "peregrinos" de serviços de saúde, não constituindo uso alternativo por dificuldades imediatamente encontradas nos serviços de atenção básica. Exemplos: "Tinha que vir no banco aí aproveitei para dar uma passada". "Fui atendida no posto da Samcil, mas não estava melhorando então resolvi vir aqui".

- Desconfiança em relação ao atendimento recebido em UBS, anseio por exames ou medicações, temor exacerbado sobre a gravidade dos sintomas

Reuniram-se nesta categoria os discursos cuja motivação para procurar atendimento no pronto-socorro era uma desconfiança em relação ao atendimento em UBS ou anseio por recursos tecnológicos. Essa categoria pode ser exemplificada com alguns dos discursos: "Aqui no pronto-socorro dão medicamento na hora, qualquer coisa faz exame". "Acompanho a pressão lá no posto, mas hoje deu vontade de vir aqui tirar a dúvida".

\section{- Falta ou férias de médicos na UBS}

Nesta categoria foram incluídas as pessoas que mencionaram a inexistência de médico na UBS no momento necessário. Exemplos: "Passei lá,

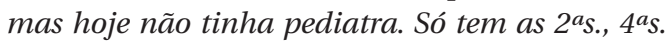
e 6 ${ }^{a}$ s.". "Não fui na UBS porque hoje não tem pediatra de manhã".

\section{- Dificuldade, incerteza de obtenção de vaga no dia}

Esta categoria engloba um conjunto de dificuldades ou obstáculos que, associados ou não à falta objetiva de vagas no dia, interpõe-se entre o usuário e o início do cuidado na UBS. Exemplos: "Não passei lá porque só atende com consulta marcada, ou então tem que chegar às 6 horas da manhã para tentar uma vaga". "Cheguei às 6 horas. No balcão mandaram esperar e depois falaram que não tinha mais vaga".

\section{- Dificuldade para agendar consulta}

Nesta categoria o discurso era de crítica às sistemáticas operacionais das UBS, que na visão do usuário criam dificuldades para o agendamento. Exemplos: "Falaram que não tinha agenda aberta para pediatria e para ir passando até abrir a agenda. Tá muito difícil...". "O acolhimento mandou marcar, aí no balcão falaram que agendamento só no dia 1o do próximo mês".

\section{- Orientações restritivas}

Discursos que fazem referências a orientações, formal ou informalmente propagadas pelos serviços, muitas vezes contraditórias, que estabele- 
Freqüência percentual das categorias discursivas dos entrevistados, segundo área/unidade básica de saúde (UBS) de procedência. Pronto-socorro Central de Itapecerica da Serra, São Paulo, Brasil, 2004

\begin{tabular}{|c|c|c|c|c|c|}
\hline Categoria discursiva & UBS A & UBS B & UBS C & UBS D & Total \\
\hline Falta de médico & 9,5 & 20,0 & 7,1 & 9,1 & 11,5 \\
\hline Vaga no dia & 49,2 & 44,0 & 42,9 & 54,5 & 47,8 \\
\hline Agendamento & 9,5 & 4,0 & 0,0 & 9,1 & 7,1 \\
\hline Orientação restritiva & 11,1 & 12,0 & 0,0 & 0,0 & 8,8 \\
\hline Organização, acesso - subtotal & 79,4 & 80,0 & 50,0 & 72,7 & 75,2 \\
\hline Uso de assistência particular, peregrino & 11,1 & 12,0 & 28,6 & 18,2 & 14,2 \\
\hline Desconfiança, anseios & 9,5 & 8,0 & 21,4 & 9,1 & 10,6 \\
\hline Outras motivações - subtotal & 20,6 & 20,0 & 50,0 & 27,3 & 24,8 \\
\hline Todas & 100,0 & 100,0 & 100,0 & 100,0 & 100,0 \\
\hline
\end{tabular}

Total dos entrevistados $=113$.

cem um padrão restritivo para o uso das UBSs. Exemplos: "A médica falou que quando é problema assim (IVAS) é para ir direto no pronto-socorro". "Lá no posto eles não atendem quando está só com dor de garganta. Falam que só é para ir se estiver passando muito mal". "O médico acha ruim, fala que emergência é no pronto-socorro”.

Visando a facilitar a comparação entre as unidades, essas seis categorias foram reunidas segundo dois grandes grupos: (1) motivações ou razões de natureza geral, relativas aos costumes de utilização de pronto-socorro não diretamente determinadas pelos problemas atuais dos serviços de atenção básica - neste grupo ( $24,8 \%$ de todas as motivações) foram incluídas as duas primeiras categorias; (2) motivações que expressam problemas no acesso e na organização dos serviços básicos - neste grupo foram incluídas as demais categorias $(75,2 \%$ das motivações).

Na Tabela 4 apresenta-se a distribuição de cada grupo de motivos das 113 pessoas entrevistadas, que procuraram atendimento no pronto-socorro por uma das hipóteses diagnósticas escolhidas, segundo a área/UBS de procedência. Pode-se observar que a média percentual de motivações atribuídas a problemas de organização e aceso das UBS é de $75,2 \%$.

A população da área/UBS C que apresentou menor freqüência de hipóteses diagnósticas escolhidas (Tabela 2) foi, também, a que relativamente apresentou menor freqüência $(50 \%)$ de procura do pronto-socorro por problemas relativos às categorias organização e acesso da UBS (Tabela 4), sendo que nas demais áreas/UBS este grupo de problemas representa de $70 \%$ a $80 \%$ das motivações.

\section{4a Etapa}

Na Tabela 5 encontram-se descritas as características de organização e acesso das UBS, verificando-se que a UBS C foi a que apresentou maior avanço no processo de mudança, superando aspectos negativos, ainda presentes nas UBS, os quais exercem papel importante no que se refere à restrição da demanda, sobretudo em relação ao atendimento de pacientes não agendados.

\section{Discussão}

Os trabalhos de análise de demanda ambulatorial e de pronto-socorro, ainda que não realizados com o objetivo específico de verificar um possível relacionamento desta demanda com a situação e condições de organização dos serviços básicos de saúde, têm apresentado como conclusões: "os dados levantados indicam que o estrangulamento de serviço de emergência se deve à desestruturação dos serviços básicos" 16 (p. 26); o pronto-socorro estudado "não é apenas um serviço de urgência, mas também, uma 'válvula de escape' dos serviços públicos de saúde...” 17 (p. 79); "um considerável número de pacientes poderia ter sido atendido nas respectivas unidades básicas, sugerindo a sua excludência dos níveis primário e secundário" 18 (p. 81).

Alguns estudos que tomaram como objeto de análise, especificamente, a demanda de unidades de emergência/urgência têm observado alta proporção de pessoas com problemas de saúde que se avaliam como passíveis de serem resolvidos mais apropriadamente em UBS 19,20,21,22,23,24. Esses estudos, embora de natureza geral sobre a 
Situação das unidades básicas de saúde (UBSs) em relação a aspectos negativos de organização e funcionamento dos serviços. Itapecerica da Serra, São Paulo, Brasil, 2004

\begin{tabular}{|c|c|c|c|c|}
\hline \multirow[t]{2}{*}{ Aspectos negativos } & \multicolumn{4}{|c|}{ Superação do negativo } \\
\hline & UBS A & UBS B & UBS C & UBS D \\
\hline $\begin{array}{l}\text { As pessoas que procuram atendimento não agendado na UBS são dispensadas com } \\
\text { base numa comunicação administrativa de capacidade esgotada, que é anunciada na } \\
\text { porta, na fila ou no balcão/recepção da unidade. Os usuários não têm a possibilidade } \\
\text { de serem atendidos para uma avaliação técnica do seu problema e são dispensados } \\
\text { sem que haja uma programação da continuidade do cuidado. }\end{array}$ & Parcial & Parcial & Sim & Parcial \\
\hline $\begin{array}{l}\text { A recepção/balcão detém o papel de controlar e decidir sobre o agendamento das } \\
\text { consultas médicas. A ação da recepção é limitada aos controles administrativos. }\end{array}$ & Parcial & Parcial & Parcial & Parcial \\
\hline $\begin{array}{l}\text { A equipe de enfermagem tem papel cuidador reduzido e sem uma participação } \\
\text { efetiva na recepção. }\end{array}$ & Parcial & Parcial & Sim & Parcial \\
\hline $\begin{array}{l}\text { O agendamento ocorre de forma a obrigar os usuários a voltarem, com ou sem } \\
\text { data estabelecida, para poder marcar uma consulta. }\end{array}$ & Não & Parcial & Sim & Não \\
\hline
\end{tabular}

demanda atendida, sugerem, também, possíveis problemas da rede básica que poderiam determinar tal característica de demanda nos serviços estudados. Entretanto, eles não tinham o objetivo de explorar retrospectivamente determinados tipos de ocorrência e relacioná-los com características da organização e do funcionamento da rede básica, de modo a propiciar instrumentos para o monitoramento.

Assim, visando a ampliar a discussão do presente trabalho, utilizou-se o recurso de um questionador fictício 25 , procurando suprir a inexistência de estudos especificamente semelhantes no que diz respeito a verificar possível relacionamento entre atendimentos desnecessários em prontos-socorros e insuficiências das UBS. Esse recurso cumpre o papel de provocar reflexões e favorecer a integração dos diversos tipos de dados coletados, segundo a estratégia de triangulação das informações recolhidas. Procurou-se, assim, evitar o parcelamento da discussão, preservando a necessária totalidade do processo crítico.

Foram três os questionamentos utilizados para organizar a discussão: (1) verificou-se que a freqüência de hipóteses diagnósticas escolhidas foi influenciada pela área de procedência das pessoas atendidas no pronto-socorro. É possível atribuir essa diferença detectada à situação de acesso e organização da atenção básica de cada UBS?; (2) o uso dessas ocorrências como instrumento para o monitoramento da atenção básica é conceitualmente coerente com os princípios das mudanças pretendidas pelo Sistema Único de Saúde (SUS)?; (3) é operacionalmente viável a utilização dessas ocorrências no monitoramento da atenção básica?

\section{1) É possível atribuir a diferença detectada das hipóteses diagnósticas escolhidas à situação de acesso e organização da atenção básica de cada UBS?}

As freqüências das hipóteses diagnósticas escolhidas variaram de $30 \%$ a 42,8\% conforme a área/ UBS de procedência considerada, sendo que as diferenças foram confirmadas como significantes pelo teste do qui-quadrado e OR. Pode-se, portanto, afirmar que houve uma associação entre as ocorrências de hipóteses diagnósticas escolhidas atendidas no pronto-socorro e as áreas/UBSs de procedência das pessoas. O desenho transversal do estudo permite constatar essa associação, mas não causalidade. Há a necessidade de ampliar o horizonte de análise. As características de organização e acesso das UBS e as motivações dos usuários para uso do pronto-socorro pelas hipóteses diagnósticas escolhidas acrescentaram elementos para que fosse discutida e evidenciada essa possível relação de causalidade.

As motivações dos usuários para a procura do pronto-socorro e os dados da observação das características das UBS são coerentes com as diferenças verificadas das freqüências das hipóteses diagnósticas escolhidas. Essa interação dos fenômenos oferece argumentos plausíveis de que a situação da atenção básica em cada área é um importante componente na determinação das ocorrências de hipóteses diagnósticas escolhidas 
no pronto-socorro. Outros autores, entretanto, sugerem que a motivação para procura do pronto-socorro não teria a ver com a situação da atenção básica, sendo antes um comportamento cultural. Kovacs et al. 26 (p. 258) afirmam: "o principal motivo para uma pessoa doente não procurar o serviço básico, onde está matriculada, é a preferência, o atrativo exercido pela unidade de maior densidade tecnológica”. Ou, ainda, “... a escolha do local de atendimento sugere que a confiança da população no serviço é diretamente proporcional à complexidade do mesmo" 16 (p. 25). No presente estudo, verificou-se que essa motivação - desconfiança em relação ao atendimento em UBSs, anseio por exames ou medicação, ou uso de equipamentos - esteve presente, entretanto, essa não foi uma razão das mais freqüentes.

Quando obstáculos objetivos de acesso à atenção básica forem removidos, o atrativo de unidades com mais equipamentos e complexidade, talvez, venha a ser mais importante na procura do pronto-socorro em substituição ao uso da UBS. Uma crença explicativa, hoje, do não uso da atenção básica pelo poder atrativo da "questão tecnológica” pode levar a destacá-la como algo independente da totalidade do funcionamento dos serviços e da vida cotidiana. Forçada essa independência da tecnologia erra-se duplamente, pois essa questão torna-se algo com uma gênese mágica e, por outro lado, retira-se da atenção básica todo o seu conteúdo valorativo, rotulando-a inelutavelmente como uma prática repetitiva de procedimentos assistenciais simplificados 27,28.

A busca irrefreável por uma consulta médica imediata por parte dos usuários não pode ser plenamente entendida e equacionada sem atentar para as construções histórico-pedagógicas envolvidas no consumo dos serviços de saúde. O fato é que historicamente se constituiu uma sensação de gravidade que está intimamente vinculada ao descaso a que os usuários dos serviços públicos de saúde foram submetidos, ela é corolário da aprendizagem da incerteza do acesso. Inverter tal significado é um dos grandes desafios do papel transformador esperançado para a atenção básica abrangente e integral.

2) O uso dessas ocorrências como instrumento para o monitoramento da atenção básica é conceitualmente coerente com os princípios das mudanças pretendidas pelo SUS?

São questões estratégicas, já amplamente reconhecidas, para a remontagem do sistema de saúde brasileiro, o papel, a extensão e a organização que a rede básica deve assumir. Desenvolver uma organização de serviços e uma prática comprometida com a integralidade do cuidado é uma questão central 29. A concretização do princípio da integralidade precisa superar o entendimento da rede básica como sinônimo de equipe mínima, simplificada, com baixa resolubilidade, que a torna incapaz de acolher as pessoas e de assumir a responsabilidade pela saúde da população de uma área, oferecendo cuidados integrais e contínuos. Estudos realizados em serviços de urgência que procuram compreender aspectos relacionados ao perfil de morbidade e motivações dessa procura têm apontado sistematicamente que parcela significativa das ocorrências poderiam ser resolvidas na atenção básica 24,30,31.

Mas, essa questão do desenvolvimento da integralidade na atenção básica, estratégica para o SUS, não seria contrária à utilização de eventos sentinela da atenção básica coletados em pronto-socorro por estes reduzirem o papel dos serviços básicos? Isto é, o instrumento em estudo não levaria a uma solução simplista relacionada apenas à existência ou não de vaga no dia, à organização de um pronto atender na unidade básica? Esse questionamento é importante de ser considerado, alerta para possíveis limitações da rede básica, mas não equaciona a necessidade e o direito das pessoas a uma resposta imediata dos serviços básicos de saúde, quando necessária, e confunde a resposta a essas necessidades com outro tipo de resposta historicamente distorcida, sobretudo da ação médica, desvirtuada nos moldes da queixa-conduta ${ }^{32}$.

Cassady et al. 33 desenvolveram um instrumento para avaliação da atenção básica, validado para o Brasil por Harzhein et al. 34, que considera o acesso, a integralidade e a continuidade dos cuidados como essenciais, incluindo como critérios a possibilidade de atendimento médico no mesmo dia, quando necessário, e o contato com o profissional que acompanha, quando possível.

Atender prontamente, e com médicos, é fundamental para a atenção básica. A questão que se antepõe, entretanto, é como fazer isso de forma racional, ampla, estável, ampliando o cuidado imediato, garantindo sua continuidade e envolvendo, quando necessário, a contribuição de variadas profissões de saúde $35,36,37$. A organização dessa atividade de pronto atender qualificada, segundo o princípio da integralidade, considerando-se a experiência acumulada da saúde pública brasileira, dificilmente surgirá subitamente, sem que se atente para o fluxo de uma longa história de unidades básicas em número insuficiente, fragilmente organizadas, capengas de recursos, embaralhadas com as pretensas verdades nacionais do Programa Saúde da Família e desumanizadas na sua ação. E este é um desafio da mudança a ser monitorado. A mudança, que não é simples nem consensual, exige canais de 
participação entre gerentes, equipes de saúde e cidadãos/usuários visando à estruturação de uma nova atenção básica marcada pela integralidade, pela humanização e pelo dinamismo na definição de suas funções 38 .

Poderia se questionar, por outro lado, que determinadas ocorrências incluídas no estudo, como hipertensão, diabetes e asma seriam situações de crises ou descompensação de doenças crônicas as quais transcenderiam, no momento da ocorrência, o papel assistencial da UBS. Esse questionamento tem certa razão factual, mas estrategicamente não deveria ser aceito, ao se pensar em uma mudança da atenção básica. Uma rota de mudança e qualificação do sistema de saúde pressupõe identificação e superação das insuficiências dos serviços. O modelo de cuidados básicos de saúde para o século 21 preconizado pela Organização Mundial da Saúde 39 fundamenta-se na equipe prestadora de cuidados de saúde, visando à sua integração em torno do doente, à prevenção, à vigilância e gestão da doença crônica.

Assim, entende-se que essas ocorrências devam ser incluídas em processos de monitoramento, apesar do seu caráter agudo, pela missão que a atenção básica tem, como princípio, para o adequado cuidado integral da saúde. Retirar as crises ou descompensações de doenças crônicas significaria deixar de obrigar que a atenção básica passe, de forma gradativa, a dar conta de um complexo mais amplo, continuado, educacional e comunicacional do cuidado das pessoas com essas morbidades 29,39,40.

3) É operacionalmente viável a utilização dessas ocorrências como indicador para apoiar o monitoramento da atenção básica?

O instrumental de monitoramento analisado não será montado sem algum esforço adicional da equipe local de saúde. Mas é algo muito sintonizado com a atividade cotidiana de gestão. A maioria dos municípios conta, por exemplo, com profissionais que assumem responsabilidades no acompanhamento das atividades de saúde integral da criança, da mulher e do adulto, que podem periodicamente viabilizar a coleta e a análise dos dados. Os profissionais que manteriam esse instrumento estariam envolvidos nessa atividade durante uma quinzena a cada trimestre, por exemplo, mas não é uma atividade distante do que fazem, é um momento de contato com a situação real, muito interessante para a observação e o reconhecimento de problemas. Tratase de uma atividade, também, pedagógica para quem se propõe a organizar programas integrais de saúde. Pisco 41 enfatiza a importância do en- volvimento dos profissionais nesses processos, na coleta e discussão dos dados, em horários regulamentares de trabalho.

O debate em torno da avaliação e do monitoramento dos serviços de saúde e da sua institucionalização tem apontado quase que invariavelmente para a inclusão da equipe de saúde nesse processo ${ }^{6}$. Conill 42 afirma, ainda, que um dos princípios a serem considerados para a implementação da política de monitoramento é a continuidade e esta depende da sustentabilidade da proposta e, neste sentido, a criação de legitimidade com adesão dos profissionais. A possível viabilidade do indicador aqui analisado não está, portanto, só no pouco tempo de trabalho exigido, mas no fato de que não constituirá um gasto de tempo paralelo às funções da equipe local de gestão ou de coordenação programática. Esses profissionais terão assim uma oportunidade de estabelecer contato mais direto com o que está acontecendo e com as dificuldades na linha da execução.

\section{Conclusões}

Os critérios utilizados na escolha das hipóteses diagnósticas escolhidas estabelecem um processo capaz de adaptar-se a distintas situações e estágios de organização da atenção básica e esta flexibilidade é uma qualidade necessária de um instrumento para monitoramento. A inclusão dos discursos dos envolvidos favorece a interpretação e a compreensão da situação sob monitoramento.

Em conclusão pode-se afirmar que: a freqüência das ocorrências escolhidas foi influenciada pelas áreas/UBS de procedência das pessoas; foi possível sugerir com razoável plausibilidade que essa influência detectada é, em parte, determinada pela situação da atenção básica em cada área/UBS; a técnica de coleta de ocorrências com características de eventos sentinela em prontosocorro para o monitoramento da atenção básica é consonante com os princípios de mudança propugnados pelo SUS; o instrumento estudado é simples, podendo ser produzido e mantido pelo gestor local; o instrumental não revela todos os aspectos e dificuldades da atenção básica, mas propicia um maior diálogo com o cotidiano dos serviços e pode somar-se a outros indicadores. Para a materialização das possibilidades desse instrumento de monitoramento será preciso desenvolvê-lo, testá-lo na prática. Ele precisa ser aprimorado, receber contribuições de novas pesquisas acadêmicas e, sobretudo, ser testado e revisado com seu uso na ação prática de monitorar. 


\section{Resumo}

O estudo tem como ponto de partida a hipótese de que determinadas ocorrências nosológicas atendidas em pronto-socorro, escolhidas com base no conceito de evento sentinela, podem estar relacionadas a falhas da atenção básica e ser utilizadas na formulação de um indicador para o monitoramento desta atenção. Foram utilizados dados quantitativos e qualitativos sobre ocorrências previamente escolhidas e atendidas em pronto-socorro. Usando-se a triangulação de métodos, verificaram-se as diferenças das freqüências dessas ocorrências entre as áreas/unidades básicas de saúde (UBS) de procedência dos pacientes e as motivações determinantes da procura pelo pronto-socorro. As freqüências das ocorrências apresentaram valores de $30 \%$ a 42,8\% conforme a área/UBS de procedência $\left(\chi^{2}=9,19\right.$ e $\left.p=0,027\right)$. As entrevistas sugeriram a existência de causalidade entre o motivo declarado da procura do pronto-socorro e a atuação das unidades básicas. Conclui-se que: (1) a freqüência das ocorrências escolhidas foi influenciada pelas áreas/UBS de procedência das pessoas; (2) essa influência decorre, em parte, da situação da atenção básica; (3) o instrumental estudado é simples e pode contribuir para o gestor local no acompanhamento cotidiano da situação dos serviços básicos.

Vigilância de Evento Sentinela; Avaliação de Serviços de Saúde; Atenção Primária à Saúde

\section{Referências}

1. Matus C. Adeus, senhor presidente: governantes e governados. São Paulo: Edições Fundap; 1997.

2. Bodstein R. Atenção básica na agenda da saúde. Ciênc Saúde Coletiva 2002; 7:401-12.

3. Ministério da Saúde. Portaria ${ }^{\circ}$. 476. Regulamenta o processo de acompanhamento e avaliação da atenção básica: pacto de indicadores da atenção básica. Diário Oficial da União 1999; 14 abr.

4. Medina MG, Aquino R, Carvalho ALB. Avaliação da atenção básica: construindo ferramentas para o SUS. Divulg Saúde Debate 2000; 21:15-28.

5. Departamento de Apoio à Descentralização, Secretaria Executiva, Ministério da Saúde. Considerações sobre a instituição de um processo de monitoramento e avaliação do SUS. Brasília: Ministério da Saúde; 2005. (Nota Técnica).

6. Felisberto E. Da teoria à formulação de uma política nacional de avaliação em saúde: reabrindo o debate. Ciênc Saúde Coletiva 2006; 11:553-63.

7. Worthen BR. Some observations about the institutionalization of evaluation. Eval Pract 1995; 16: 29-36.

\section{Colaboradores}

P. T. Puccini realizou levantamento e análise dos dados e elaborou o artigo. V. K. Cornetta realizou revisão crítica do artigo.
8. Hartz ZMA. Institucionalizar e qualificar a avaliação: outros desafios para a atenção básica. Ciênc Saúde Coletiva 2002; 7:419-21.

9. Penna MLF. Condição marcadora e evento sentinela na avaliação de serviço de saúde. In: Santana JP, organizador. Desenvolvimento gerencial de unidades básicas do Sistema Único de Saúde. Brasília: Organização Pan-Americana da Saúde; 1997. p. 121-8.

10. Novaes HMD. Avaliação de programas, serviços e tecnologias em saúde. Rev Saúde Pública 2000; 34:546-59.

11. Deslandes SF. Concepções em pesquisa social: articulações com o campo da avaliação em serviços de saúde. Cad Saúde Pública 1997; 13:103-7.

12. Conill EM. Complementando a discussão sobre a atenção básica: podem o acesso, a integralidade e o controle social se constituírem em temáticas de consenso para a avaliação da reforma brasileira? Ciênc Saúde Coletiva 2002; 7:421-3. 
13. Puccini PT. Análise de instrumentos de apoio ao monitoramento dos serviços de atenção básica [Tese de Doutorado]. São Paulo: Faculdade de Saúde Pública, Universidade de São Paulo; 2005.

14. Organização Mundial da Saúde. Classificação estatística internacional de doenças e problemas relacionados à saúde, 10a revisão. São Paulo: Centro Colaborador da OMS para a Classificação de Doenças em Português; 1995.

15. Bardin L. Análise de conteúdo. Lisboa: Edições 70; 2004.

16. Martins NGR, Paskulin LMG. Emergência: fatores que levam a população infantil a recorrer a este serviço. Rev Bras Enferm 1992; 45:21-7.

17. Salla J, Ghellar M, Kaufman ML, Ilha NS, Frandoloso PR. Perfil da demanda de um serviço de prontosocorro, Santa Maria. Saúde (Santa Maria) 1989; 15:71-80.

18. Gutierrez MR, Barbieri MA. Sistema Único de Saúde e demanda ambulatorial: os pacientes do Hospital das Clínicas da Faculdade de Medicina de Ribeirão Preto - USP. Medicina (Ribeirão Preto) 1998; 31:81-98.

19. Yamada ATT, Castro CGJ, Almeida MF, Nakazaki RMD, Garbin W, Sá ENC, et al. Estudo do perfil da demanda do serviço de pronto-socorro do Hospital Geral de Pedreira: relatório. São Paulo: Secretaria Estadual de Saúde de São Paulo/Faculdade de Saúde Pública, Universidade de São Paulo; 2000.

20. Yamada ATT, Castro CGJ, Almeida MF, Nakazaki RMD, Garbin W, Sá ENC, et al. Estudo do perfil da demanda do serviço de pronto-socorro do Hospital Geral de Grajaú: relatório. São Paulo: Secretaria Estadual de Saúde de São Paulo/Faculdade de Saúde Pública, Universidade de São Paulo; 2001.

21. Castro CGJ, Almeida MF, Cardoso MRL, Bertolino ME, Puccini PT. Estudo do perfil da demanda do serviço de pronto-socorro do Hospital Geral de Itapecerica da Serra. São Paulo: Secretaria Estadual de Saúde de São Paulo/Faculdade de Saúde Pública, Universidade de São Paulo; 2001.

22. Yamada ATT, Castro CGJ, Almeida MF, Garbin W, Sá ENC, Gomes MC. Estudo do perfil da demanda do serviço de pronto-socorro do Hospital Geral de Itaquaquecetuba: relatório. São Paulo: Secretaria Estadual de Saúde de São Paulo/Faculdade de Saúde Pública, Universidade de São Paulo; 2002.

23. Castro CGJ, Almeida MF, Yamada ATT, Garbin W, Oliveira NA, Oliveira MS, et al. Estudo do perfil da demanda do serviço de pronto-socorro do Hospital de Itaim Paulista: relatório. São Paulo: Secretaria Estadual de Saúde de São Paulo/Faculdade de Saúde Pública, Universidade de São Paulo; 2002.

24. Mendes HWB. Regionalização da assistência à saúde: análise de demanda ao serviço de urgência/emergência de um hospital universitário [Tese de Doutorado]. São Paulo: Faculdade de Saúde Pública, Universidade de São Paulo; 2003

25. Donato AF. Traçando redes de comunicação: releitura de uma práxis da educação no contexto da saúde [Tese de Doutorado]. São Paulo: Faculdade de Saúde Pública, Universidade de São Paulo; 2000
26. Kovacs MH, Feliciano KV, Sarinho SW, Veras AA. Acessibilidade às ações básicas entre crianças atendidas em serviços de pronto-socorro. J Pediatr (Rio J) 2005; 81:251-8.

27. Vázquez AS. Filosofia da práxis. 4a Ed. São Paulo: Editora Paz e Terra; 1990.

28. Kosik K. Dialética do concreto. São Paulo: Editora Paz e Terra; 1995.

29. Gomes MCPA, Pinheiro R. Acolhimento e vínculo: práticas de integralidade na gestão do cuidado em saúde em grandes centros urbanos. Interface Comun Saúde Educ 2005; 9:287-301.

30. Furtado BMASM, Araújo Jr. JLC, Cavalcanti P. O perfil da emergência do Hospital da Restauração: uma análise dos possíveis impactos após a municipalização dos serviços de saúde. Rev Bras Epidemiol 2004; 7:279-89.

31. Gérvas J. Cuidados primários de saúde na Europa: tendências atuais. Rev Bras Epidemiol 2004; 7:350-69.

32. Gonçalves RBM. Tecnologia e organização social das práticas de saúde. São Paulo: Editora Hucitec/ Rio de Janeiro: ABRASCO; 1994.

33. Cassady CE, Starfield B, Hurtado MP, Berk RA, Nanda JP, Friedenberg LA. Measuring consumer experiences with primary care. Pediatrics 2000; 105(4 Pt 2):998-1003.

34. Harzheim E, Starfield B, Rajmil L, Álvarez-Dardet C, Stein AT. Consistência interna e confiabilidade da versão em português do Instrumento de Avaliação da Atenção Primária (PCATool-Brasil) para serviços de saúde infantil. Cad Saúde Pública 2006; 22:1649-59.

35. Bosi MLM, Uchimura KY. Avaliação da qualidade ou avaliação qualitativa do cuidado em saúde? Rev Saúde Pública 2007; 41:150-3.

36. Takemoto MLS, Silva EM. Acolhimento e transformações no processo de trabalho de enfermagem em unidades básicas de saúde de Campinas, São Paulo, Brasil. Cad Saúde Pública 2007; 23:331-40.

37. Camelo SHH, Angerami ELS, Silva EM, Mishima SM. Acolhimento à clientela: estudo em unidades básicas de saúde no Município de Ribeirão Preto. Rev Latinoam Enferm 2000; 8:30-7.

38. Puccini PT, Cecílio LCO. A humanização dos serviços e o direito à saúde. Cad Saúde Pública 2004; 20:1342-53.

39. Chronic Diseases and Health Promotion Department, Noncommunicable Diseases and Mental Health Cluster, World Health Organization. Preparing a health care workforce for the 21st century: the challenge of chronic conditions. http://www. who.int/chp/knowledge/publications/workforce report.pdf (acessado em 16/Mai/2007).

40. Sala A, Nemes MIB, Cohen DD. Metodologia de avaliação do trabalho na atenção primária à saúde. Cad Saúde Pública 1998; 14:741-51.

41. Prisco LA. A avaliação como instrumento de mudança. Ciênc Saúde Coletiva 2006; 11:566-8.

42. Conill EM. Sobre a importância da parcimônia, do diálogo e da continuidade para a institucionalização da avaliação na atenção básica. Ciênc Saúde Coletiva 2006; 11:564-6.

Recebido em 06/Jul/2006

Versão final reapresentada em 28/Jan/2008

Aprovado em 15/Fev/2008 